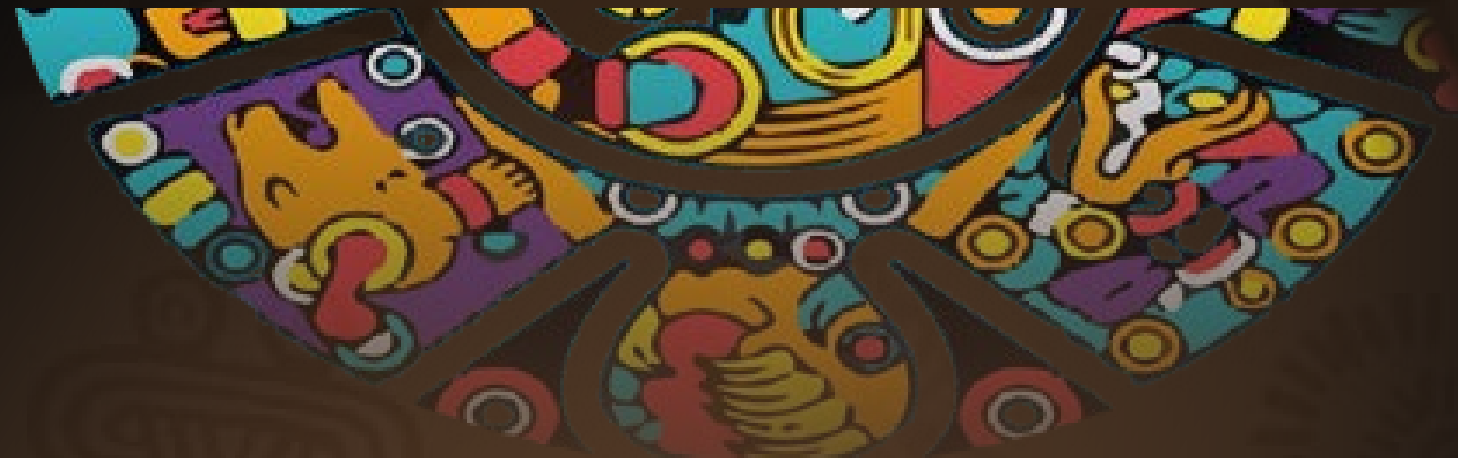

\title{
¿QUÉ SIGNIFICA EL BICENTENARIO DE LA INDEPENDENCIA DE CENTROAMÉRICA?
}

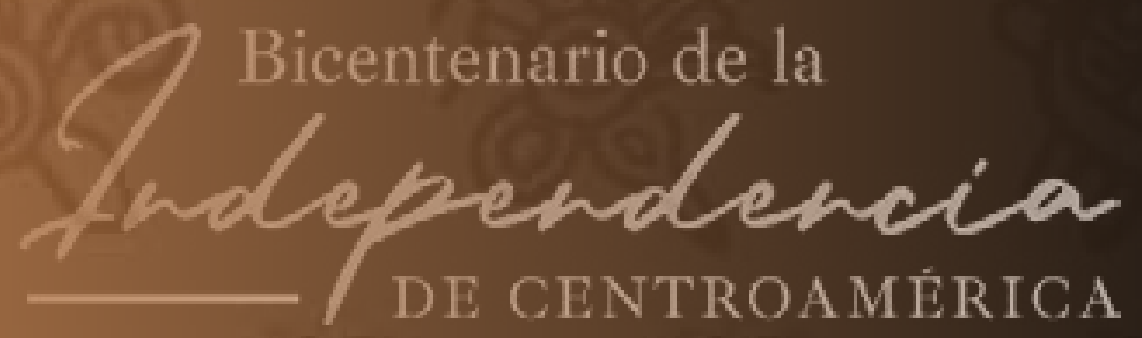




\section{¿Qué significa el Bicentenario de la independencia de}

\section{Centroamerica?}

What does the Bicentennial of the Independence of Central America?

\section{Juana Gricelda Tellez Vado}

Departamento de Antropologia

Facultad de Humanidades y Ciencias Jurídicas

Universidad Nacional Autónoma de Nicaragua

UNAN-Managua

ID Orcid https://orcid.org/0000-0003-2810-0110

jugrictelva@gmail.com
Recibido: 04-09-2021

Aceptado: 10-09-2021

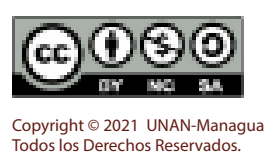

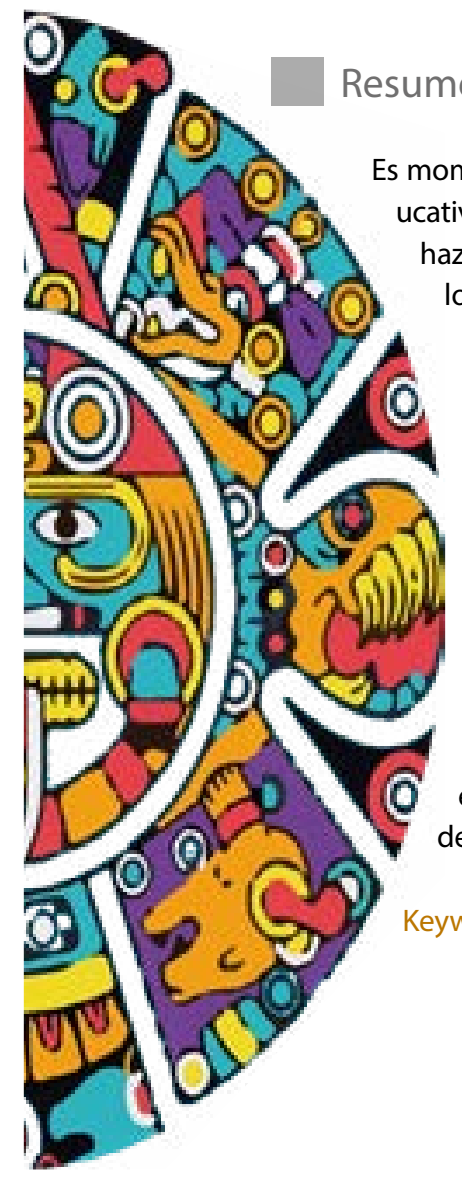

Palabras claves: antropologia, bicentenario, cultura

\section{Abstract}

It is time to rekindle the alert to strengthen and deepen, through educational exercise, cultural resistance, which has made us strong and achieve successes that seem impossible to achieve, defending the country from the coup attempt promoted by the serfs and vassals of the conquerors, reactivating the economy, production and promoting technological innovation, fighting the pandemic. We are a small town but one that knows where it is going and how to fight.

Keywords: anthropology, bicentennial, culture 


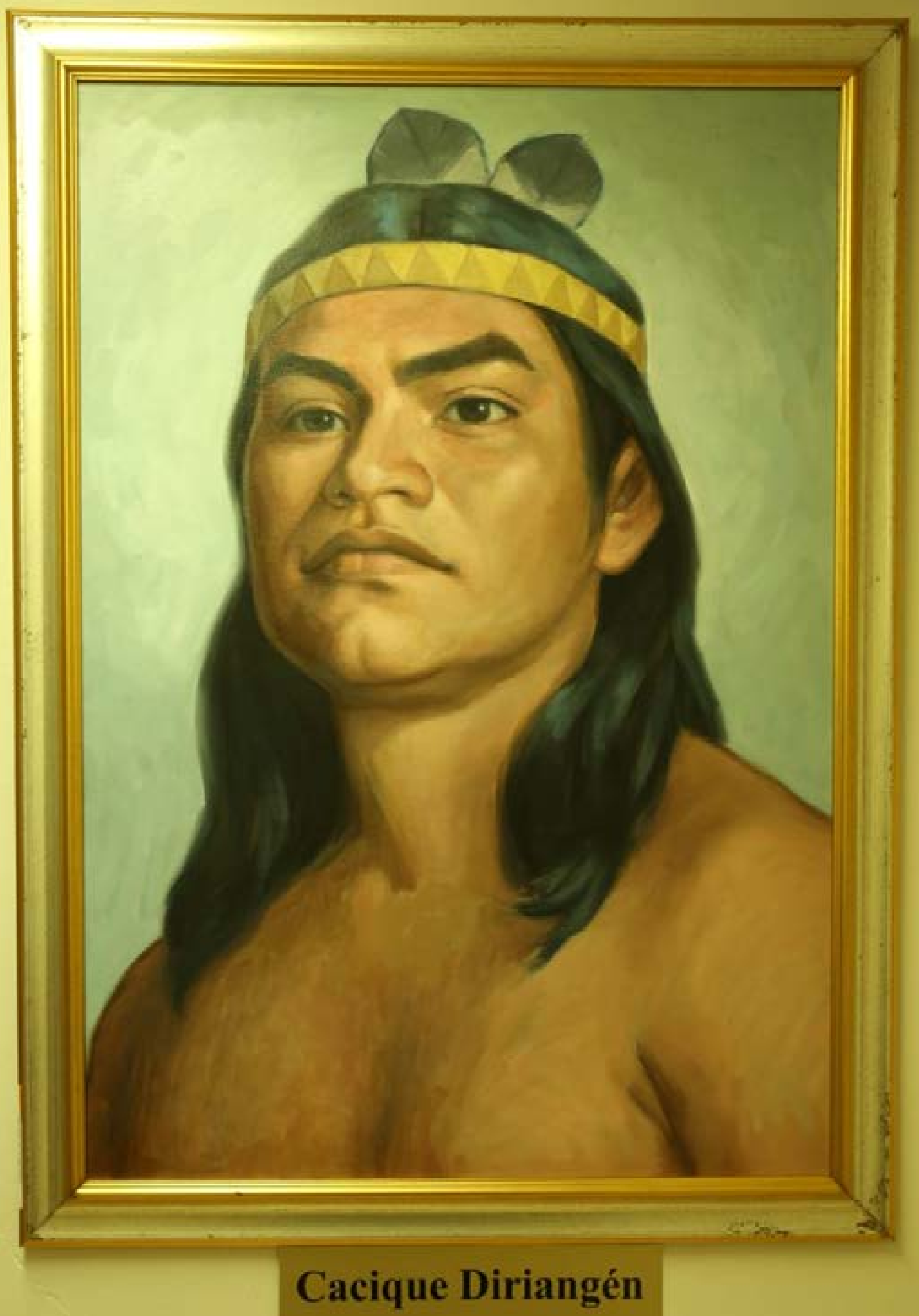




\section{Introducción}

Cada 15 de septiembre recordamos, reflexionamos y buscamos una mejor coomprensión sobre su significado. El 200 aniversario de la memorable primera independencia de Nicaragua es una oportunidad, que nos fascina, para comprender esos procesos en que nuestros pueblos originarios y sus descendientes forjaron, impulsaron y llegaron a ese hito tan importante en nuestra historia.

Son procesos que guardan las claves, las pistas, de las batallas, las campañas heroicas en favor de la emancipación marcada en 300 años de lucha previas a la independencia de la Región Centroamericana, a través de la resistencia heroica que se inició con las batallas que le dio el Cacique Diriangén, que se negó a rendirse, "dialogó con los españoles, con los invasores, dialogó con ellos y le quisieron imponer sus condiciones y Diriangén no las aceptó y los combatió, los enfrentó en una batalla desigual", y los 300 años de lucha para llegar a esa declaración de independencia, conllevó a resistir un total de 500 años de lucha para forjar la autodeterminación y la independencia de nuestros pueblos" (Barbosa Miranda, 2007).

Es conveniente realizar una pausa a la narrativa histórica, para apoyarnos en un pilar que ha estado presente en la historia de la humanidad, y es que la cultura independiente de las épocas en que se le situé, e independiente del nivel de desarrollo del conocimiento "la cultura, al igual que la dignidad es una condición inseparable del ser humano".

Lo anterior explica y nos ayuda a la coomprensión del auto-reconocimiento de los pueblos que fueron dueños de sus tierras, de sus culturas, sus lenguas, su sistema de creencias, de su historia, sus batallas militares que dieron inicio en 1523 entre el ejército invasor del conquistador español, Gil González, y un ejército indígena dirigido por Diriangén; el reconocimiento a los indígenas nicaragüenses que desde antes de la llegada de los españoles tenían ya una estructura social con carácter de organización militar y resolvían sus diferencias domesticas originadas por diferentes causas, lo que les había permitido construir armas rudimentarias, ejercitar tácticas de guerra en sus combates y fundamentar espiritualmente la esencia y concepciones de las guerras que formaban parte de su vida (Barbosa Miranda, 2007), lo cual tenía gran influencia en su cultura y tradiciones de resistencia.

En unas guerras en las que nuestros ancestros libraban con inferiores recursos materiales y tecnológicos muy grandes, la valentía, la moral y la convicción eran un plus aportado por el sentido de identidad, apropiación de nuestras raíces generando la resistencia cultural.

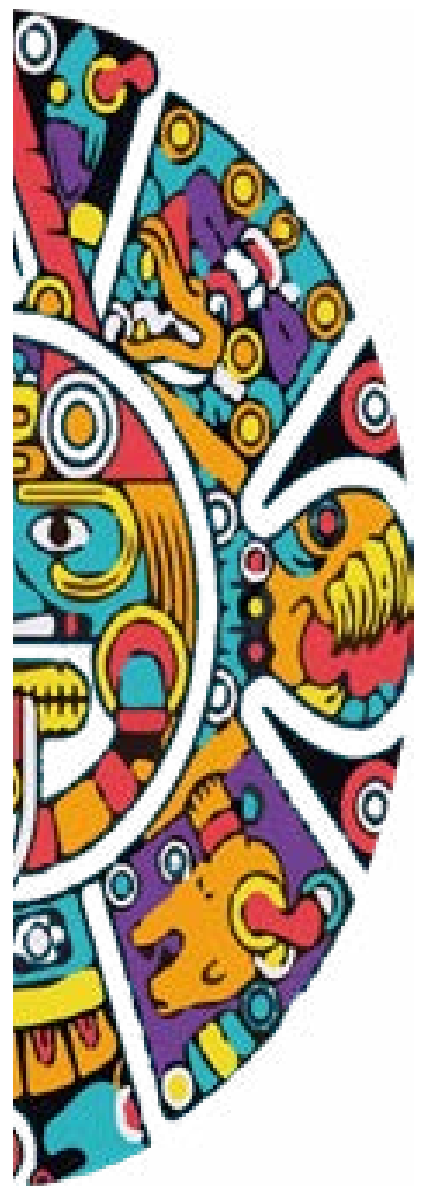




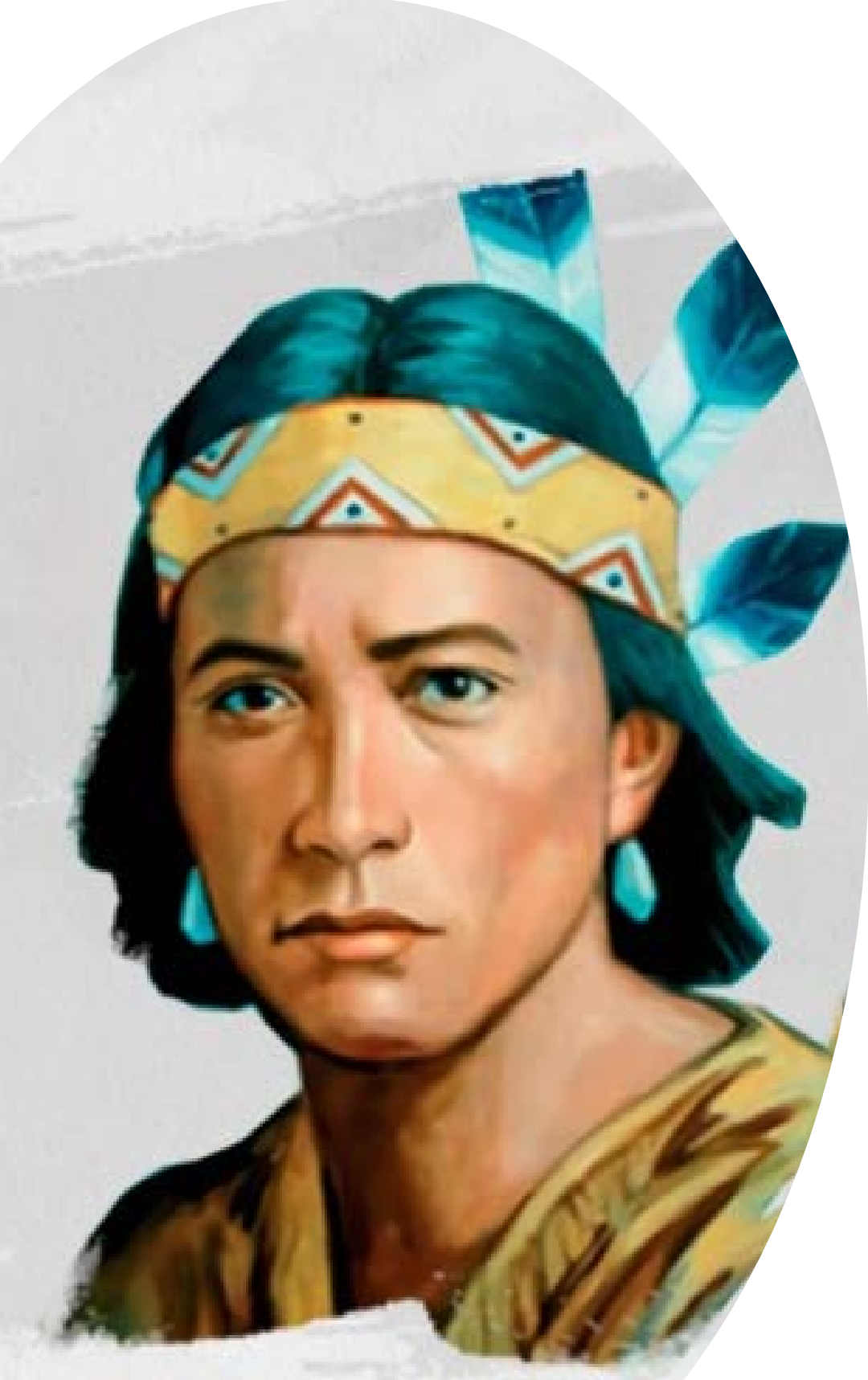

Estas ideas tratan de poner en relieve, más allá de lo anecdótico, los elementos de primer orden que constituyen la cultura y que dieron el sentido que explican la lógica de la resistencia, negra, indígena y mestiza. Desde la perspectiva institucional-cultural se enfati-zan las tradiciones históricas y culturales del sistema político administrativo. La estabilidad intra e inter or $\neg$ ganizacional producto de la cultura organizacional homogénea en la cual los actores realizan sus activi $\neg$ dades colectivas y logran sus objetivos comunes como es el acceso a la educación, el derecho a la tierra, el medio ambiente, los derechos del trabajo, los derechos humanos; la discriminación racial, la calidad de vida de las mujeres, la continuidad del sistema colonial impuesto en el continente, hace más de cinco siglos, basado en los paradigmas del patriarcado y el colonialismo interno como ejes de dominación que prolongan las elites modernas, en la sobrevivencia de formas de discriminación que no solamente plantean discriminaciones, tensiones y desencuentros interculturales, sino que impactan fundamentalmente en las mujeres afectando su calidad de vida y su acceso a los derechos humanos y ciudadanos fundamentales. (Elizabeth, 2004). 


\section{Conclusión}

A doscientos años el desafío continuó, los españoles conquistadores, so pretexto, del robo, y el exterminio de toda una cultura justificaron así sus fechorías:"Tierra de Nadie" (res nullius), principio que supuso de hecho y derecho el reparto en ocasiones del territorio entre estados europeos a través de la ocupación, por desconocimiento de la propiedad indígena cuando la hubiese (Lemoine, 2003)

En la actualidad los conquistadores norteamericanos, so pretexto, de intervenir política, económica y culturalmente justifican sus fechorías basadas en la siguiente falacia: "Ninguna nación tiene el derecho de expulsar a otra, si no es por un designio especial del cielo como el que tuvieron los israelitas, a menos que los nativos obraran injustamente con ella. En este caso tendrán derecho a librar, legalmente, una guerra con ellos y a someterlos" (Lemoine, 2003) Les molesta que vivamos a nuestra manera, que alcancemos el desarrollo con justicia y equidad, nos quieren seguir prohibiendo vivir a nuestro modo y manera, nos quieren volver a negar el derecho de ser".

Es momento de reavivar la alerta para fortalecer y profundizar, por medio del ejercicio educativo, la resistencia cultural, que nos ha hecho fuertes y alcanzar éxitos que parecen hazañas imposibles de lograr, defendiendo el país del intento golpista impulsado por los siervos y vasallos de los conquistadores, reactivando la economía, la producción e impulsando la innovación tecnológica, combatiendo la pandemia. Somos un pueblo pequeño pero que sabe hacia dónde va y como luchar.

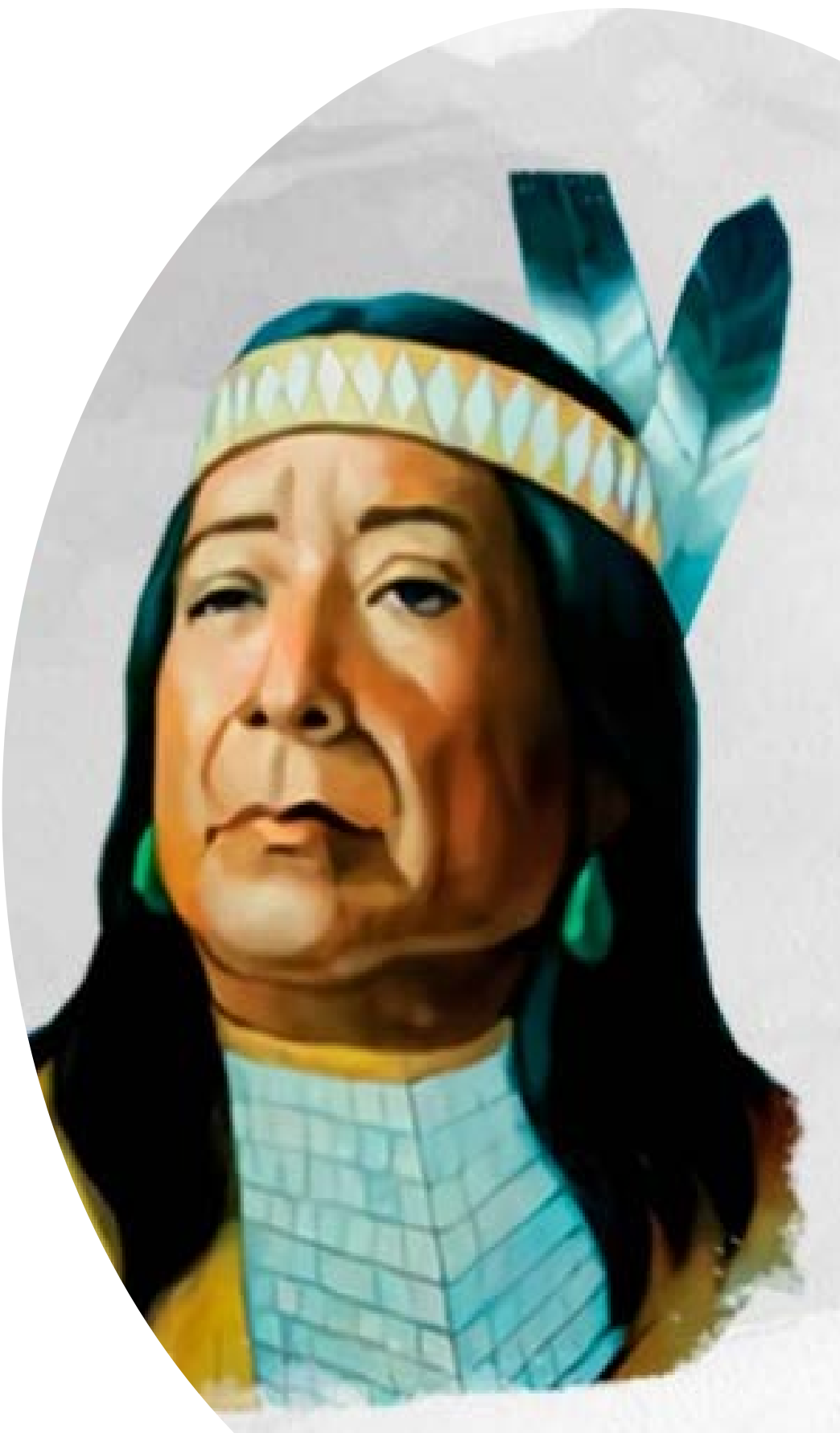




\section{Raíces}

Revista Nicaragüense de Antropología Año 5 | 2021 Edicion Especial -Bicentenario

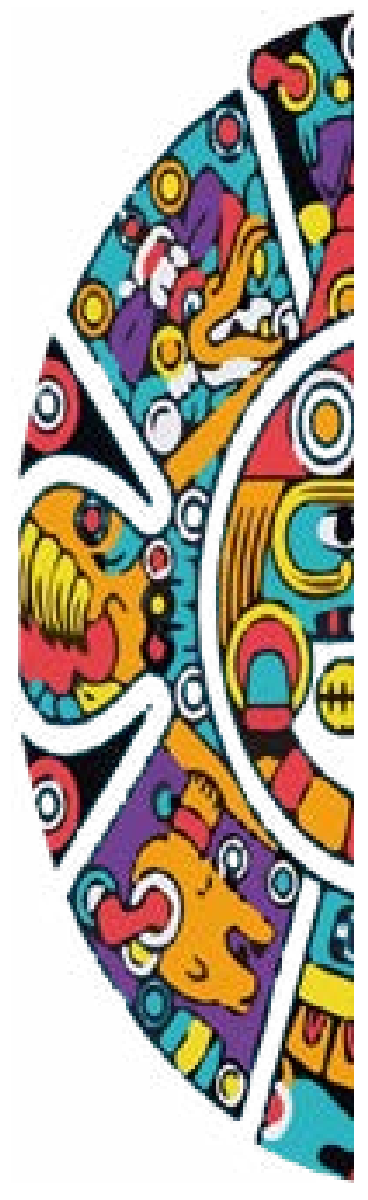

Bibliografía

Barbosa Miranda, F. (2007). Síntesis de la Historia Militar de Nicaragua de las guerras intertribales precolombinas al Ejército de Nicaragua (antes de 1523 a 2005). Managua: Centro de Historia Militar-Ejercito de Nicaragua.

Elizabeth, P. B. (2004). Una aproximación a la problemática de género y etnicidad en América Latina. CEPAL - SERIE Mujeres y desarrollo - № 53, 1 - 73.

Lemoine, M. (2003). En nombre del "destino manifiesto". Cono Sur, No 47, 20-21.

Molano L., O. L. (2007). Identidad cultural un concepto que evoluciona. Opera, núm. 7, Bogotá, Colombia, 69-84.

Juana Gricelda Téllez Vado

Licenciatura en Antropología Social en la Universidad Nacional Autónoma de Nicaragua (UNAN-MANAGUA). Maestría en Antropología y Liderazgo Social en la Universidad Nacional Autónoma de Nicaragua (UNAN-MANAGUA). Conocimientos y experiencia en tareas de acompañamiento y asistencia técnica- metodológica a prácticas de Familiarización, prácticas de Especialización. Desarrollo de temáticas en los contextos rurales y urbanos concernientes dinámicas familiares en relación a la familia, parentesco, relaciones y roles de género; familia - comunidad y territorio. Habilidades para el trabajo interdisciplinario, el manejo de relaciones interpersonales y el manejo de grupos. Interés por la actualización profesional. Diseño y análisis y diseños de investigación. 